\title{
TEAPOT: A THIN-ELEMENT ACCELERATOR PROGRAM FOR OPTICS AND TRACKING
}

\author{
L. SCHACHINGER and R. TALMAN \\ SSC Central Design Group, c/o Lawrence Berkeley Laboratory, Berkeley, \\ CA 94720
}

(Received July 24, 1986; in final form December 1, 1986)

The program TEAPOT is intended for fast and exact particle tracking in an accelerator with magnet errors and misalignments. The formalism uses only thin elements, but, unlike other "kick" codes, TEAPOT tracking is exact to all orders in all six phase-space displacements. This procedure of exact tracking in an approximate lattice can be contrasted with approximate tracking in an "exact" lattice, as is performed in existing programs. TEAPOT also has provisions for a full Twiss analysis after errors and misalignments have been added and for the compensation of error-induced coupling, tune-shifts, and chromaticity shifts.

\section{INTRODUCTION}

Certain nonlinear particle-tracking problems are greatly simplified if the accelerator lattice consists only of drift sections and thin elements. These simplifications include preserving symplecticity, handling momentum offsets, and handling magnet errors and misalignments. Though somewhat idealized, such a lattice can be regarded as containing the essence of a more realistic thick-element lattice. The correspondence can be arbitrarily improved by subdividing the elements. Since momentum offsets are handled exactly, TEAPOT is exact for nonrelativistic machines, as well as for highly relativistic ones. The purpose of the program TEAPOT described in this paper is to exploit these simplifications for fast and exact tracking in the presence of various accelerator errors. This exactness in particle tracking will be demonstrated and contrasted with other standard codes.

The more basic formulas to be used are spelled out in this introduction, and the gross geometry of the central orbit is described in Section 2. One problem is that of mechanical translation of a realistic thick-element lattice (Standard Input Format ${ }^{1}$ ) into a thin-element lattice whose properties agree acceptably with those of the original. This is described in Section 3. Another problem is tracking a particle through the thin-lens lattice. This is described in Section 4. Analysis and retuning of the perturbed lattice is described in Section 5. Section 6 describes some of the operations which TEAPOT can be instructed to perform. Section 7 contains a comparison of the performance of various programs on a sample lattice. This discussion relies heavily on the work of Forest, described in Ref. 2 and shows that TEAPOT can legitimately be called a symplectic integrator.

By a thin element we mean a magnetic multipole of infinitesimal thickness $L$. 
Its only nonvanishing components are assumed to be $B_{x}$ and $B_{y}$, where the local coordinate system $x, y, s$ has its $s$ axis normal to the multipole plane. The fields depend only on $x$ and $y$ and are given explicitly by the equation

$$
L\left(B_{y}+i B_{x}\right)=L B_{0} \sum_{n=0}^{M}\left(b_{n}+i a_{n}\right)[(x-\Delta x)+i(y-\Delta y)]^{n}
$$

where $B_{0}$ is the nominal dipole magnetic field and $\Delta x$ and $\Delta y$ are displacements of the multiple from its nominal position. The multipole coefficients $b_{n}$ and $a_{n}$ in Eq. (1) are conventional (e.g., Ref. 3). The index of the highest nonvanishing element is $M$. For a given multipole, the dipole element $B_{0} b_{0}$ may or may not vanish, but it will be assumed for the reference orbit that $a_{0}=0$ always (no "out-of-plane" bends in the design orbit). The restriction to "in-plane" bends is easily removed. This will be done in a version of the program called TEAPOTV, which will, as a result, be somewhat slower.

The particle energy, momentum, and velocity are related by

$$
\mathbf{p}=\frac{E \mathbf{v}}{c^{2}} .
$$

In a pure magnetic field $E,|\mathbf{v}|$ and $|\mathbf{p}|$ are constant. The momentum deviation from a central momentum $p_{0}$ is described by $\delta$, where

$$
p=p_{0}(1+\delta) \text {. }
$$

It should be recognized that $\delta$ is introduced for reasons of tradition only. It is not an expansion parameter and is never required to be small in TEAPOT. For this report, $\delta$ will be assumed to be constant, but a version of the program called TEAPOTS, also exact, handles synchrotron oscillations in which $\delta$ varies. In particle tracking, path length differences are calculated and $\delta$ is changed appropriately on passage through accelerating cavities.

The equation of motion for a particle is

$$
\frac{d \mathbf{p}}{d t}=\frac{E}{c^{2}} \frac{d \mathbf{v}}{d t}=e \mathbf{v x B}=e\left|\begin{array}{ccc}
\hat{x} & \hat{y} & \hat{s} \\
v_{x} & v_{y} & v_{s} \\
B_{x} & B_{y} & 0
\end{array}\right|,
$$

where $v_{x}, v_{y}$, and $v_{s}$ are velocity components in the local frame. To exploit the infinitesimal thickness in the $s$ direction, it is useful to regard $s$ rather than $t$ as the independent variable. They are related by

$$
d t=\frac{d s}{v_{s}},
$$

whereupon Eq. (4) yields

$$
\begin{aligned}
& \frac{d v_{x}}{d s}=-\frac{e v}{p} B_{y}, \\
& \frac{d v_{y}}{d s}=\frac{e v}{p} B_{x} .
\end{aligned}
$$


Treating the force as an impulse, we obtain the fractional velocity changes

$$
\begin{aligned}
\frac{\Delta v_{x}}{v} & =-\frac{1}{1+\delta}\left(\frac{B_{y} L}{p_{0} / e}\right), \\
\frac{\Delta v_{y}}{v} & =\frac{1}{1+\delta}\left(\frac{B_{x} L}{p_{0} / e}\right) .
\end{aligned}
$$

Each quantity in parenthesis has been grouped for convenience with units. It is the ratio of the "field integral" measured in tesla-meters to the $B \rho$ value, according to which $p_{0} / e$ is also expressed in tesla-meters. In TEAPOT the actual multipole coefficients are stored in the form

$$
\begin{aligned}
& \tilde{a}_{n}=\frac{L B_{0} a_{n}}{p_{0} / e}, \\
& \tilde{b}_{n}=\frac{L B_{0} b_{n}}{p_{0} / e} .
\end{aligned}
$$

The component $v_{s}$ can always be recovered using the requirement that $|\mathbf{v}|$ is constant:

$$
\begin{aligned}
\left(\frac{v}{c}\right)^{2} & =\left(\frac{v_{x}}{c}\right)^{2}+\left(\frac{v_{y}}{c}\right)^{2}+\left(\frac{v_{s}}{c}\right)^{2} \\
& =c^{2} \frac{p^{2}}{E^{2}} \\
& =\frac{1}{1+\frac{m_{0}^{2} c^{4}}{p_{0}^{2} c^{2}(1+\delta)^{2}}}
\end{aligned}
$$

where $m_{0}$ is the particle rest mass. For all cases presently foreseen, it would be reasonable to approximate the velocity always by $c$, but the attempt will be made to write formulas which are valid even in less highly relativistic situations. We do however work with velocity ratios $v_{x} / c, v_{y} / c, v_{s} / c$.

A critical issue for a program such as this is its freedom from bugs. As with any new and complicated computer program it is hard to prove that TEAPOT is free of them, though many checks have been performed. It can be claimed that the innermost tracking loop (enclosed in boxes in Section 4) is sufficiently short and has been subjected to enough tests to have a high probability of being correct. It consists of only some 60 lines of FORTRAN code.

A possible eventual development would be to code this tracking section for a special-purpose computer, for investigating questions of long-term stability. Short of this, this section of code could perhaps be further optimized by handwritten assembler language or the use of fixed-point variables. In such a development, speed would be of the essence. For the SSC, a version of this code is being developed for special-purpose simulation of operational issues like injection and energy ramping.

When we say that particle tracking is exact, we mean that, within the precision 
of the computer being used, including round-off errors, and with the assumption that all elements are exactly described by expansions of the form given by Eq. (1), the particle orbits are faithfully followed. That being the case, one can inquire whether the tracking has the admirable property of being symplectic. Technically, symplecticity refers to a Hamiltonian formalism in which $x, p_{x}$, etc., are used. Since, in this report, the Lagrangian variables $x, d x / d t=v_{x}$, etc., are used, the code cannot, technically be called symplectic. This can be regarded as a quibble, however, as canonical variables can be reconstructed at will. Another way in which the code is superficially not symplectic results from the fact that, for the version described in this report, only transverse coordinates are propagated. While a symplectic four-dimensional formalism is possible, the mixing of transverse and longitudinal coordinates, say, in a bend or even in a coordinate rotation, makes it likely for local symplecticity to be violated. Again, this effect could be removed in the 4-D formalism with care and is, indeed, removed in the 6-D formalism of TEAPOTS, which faithfully tracks both transverse and longitudinal coordinates. That program can be said to be symplectic in six dimensions if the above-mentioned distinction between Lagrangian and Hamiltonian coordinates is ignored. Notice that it is not that this is a so-called "kick code" which makes it symplectic, but that it is an exact kick code.

Another desirable feature of a tracking program is that it be a "symplectic integrator". TEAPOT can be regarded as a symplectic integrator of lowest order. (Since higher-order symplectic integrators entail drift spaces of negative length, ${ }^{5}$ they do not satisfy the prejudices of the authors that the representation should be "physical" in the sense elaborated on in Section 4.3).

Again, since the equations are exact, it is superfluous to check that a one-turn map (in appropriate variables) is symplectic, but that has been done numerically and found to be true. This can be regarded as a check of the claim that the equations are exact (necessary, but of course not sufficient.)

In the abstract, these features have been codified by the statement that TEAPOT performs exact tracking in an approximate lattice, in contrast to existing programs which perform approximate tracking in an "exact" lattice.

\section{GROSS GEOMETRY OF THE CENTRAL ORBIT}

Our coordinate conventions conform to MAD. ${ }^{4}$ Along with the strengths defined in Eq. (8) and offsets $\Delta x$ and $\Delta y$ defined in Eq. (1), the $N+1$ multipoles making up the lattice are described by the absolute Cartesian coordinates

$$
X_{i}, Y_{i}=0, Z_{i} \quad(i=0, \ldots, N) .
$$

It is assumed that

$$
X_{0}=Z_{0}=X_{N+1}=Z_{N+1}=0
$$

and that

$$
X_{1}=0 \text {, }
$$

which correspond, in words, to the assumption that there is a null element at the 


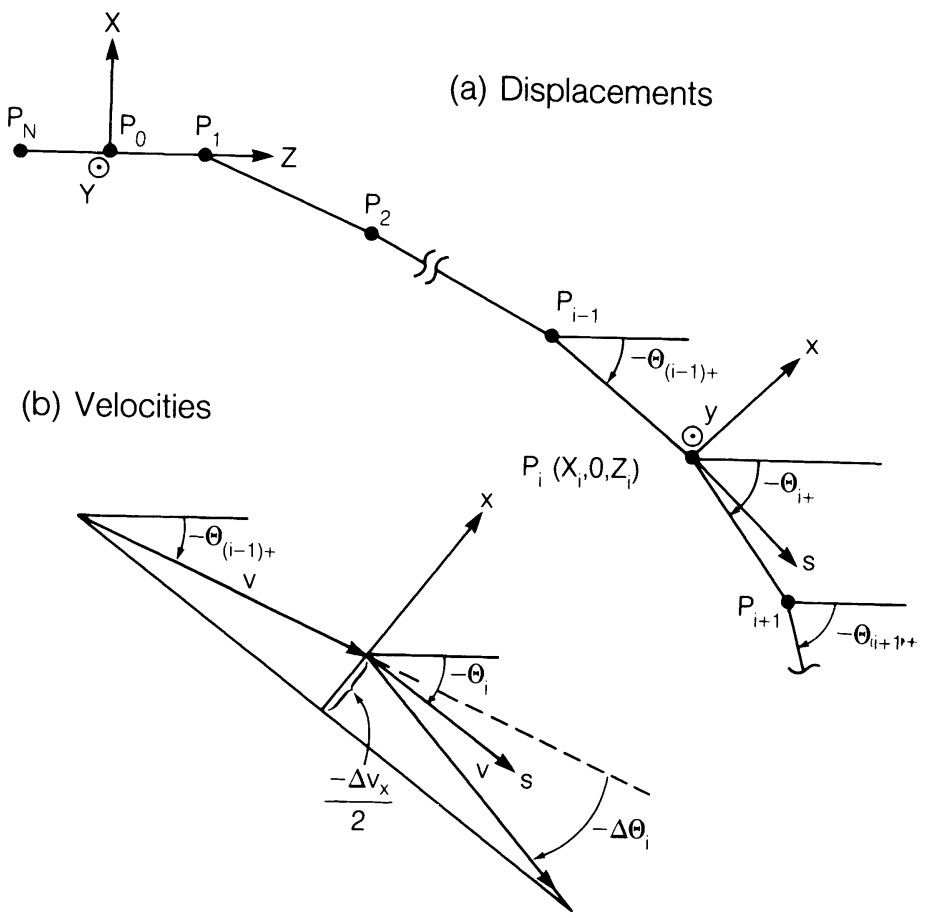

FIGURE 1 Gross geometry. Quantities labeled with negative signs are numerically negative for the configuration illustrated. This is also indicated by the arrow pointing in the negative (i.e., clockwise) direction.

origin and the central orbit at that point passes through the origin parallel to the $Z$ axis. At that point, the $s$ and $Z$ axes coincide. The entire central orbit lies in the $Y=0$ plane.

The direction of the central orbit is specified by

$$
\Theta_{i+}(i=0, \ldots, N),
$$

which is its angle counter-clockwise from the $Z$ axis as it leaves the $i$ th element. The gross geometry is indicated in Fig. 1.

For a given multipole, the dipole element $\tilde{b}_{0}$ may or may not vanish. In any case, there is a required relationship between $\tilde{b}_{0}$ and the angular deflection $\Delta \Theta$ suffered by the nominal central orbit at the element. From Figure 1 and Eqs. (7) and (8), the relationship is

$$
\begin{aligned}
\Delta \Theta_{i} & =2 \sin ^{-1} \frac{1}{2} \frac{\Delta v_{x}}{v} \\
& =-2 \sin ^{-1} \frac{\tilde{b}_{0}}{2} .
\end{aligned}
$$

The sign conventions are such that a positive particle in a positive dipole field follows a clockwise path when viewed from above (i.e., from a point with $y$ positive.) 


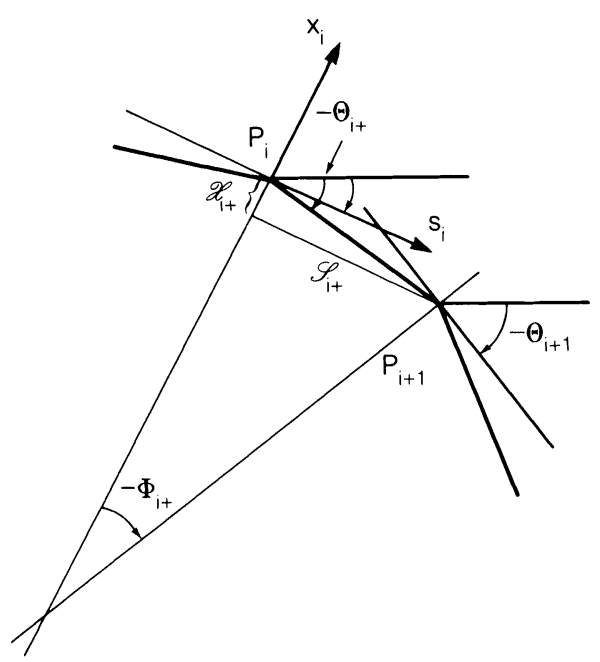

FIGURE 2 Angular relationship between frame $\mathscr{P}_{i+1}$ and $\mathscr{P}_{i}$. The significance of negative labels is the same as in Fig. 1.

When the multipole includes a kink in the central orbit, care must be exercised in defining the multipole plane as the bisector of the initial and final directions. (Also, path lengths through thin and thick elements will differ slightly.) Hence, we have

$$
\begin{gathered}
\Theta_{0}=0 \\
\Theta_{i}=\frac{1}{2}\left(\Theta_{(i-1)+}+\Theta_{i+}\right)(i=1, N) .
\end{gathered}
$$

These angles satisfy

$$
0 \geq \Theta_{i} \geq \Theta_{i+1} \geq-2 \pi(i=0, N),
$$

assuming, as we will, that there are no negative bends.

Certain calculations can be performed in advance on the equilibrium orbit which speed up the tracking of particles. These are indicated in Fig. 2. The quantities $\mathscr{X}_{i+}, \mathscr{S}_{i+}$ are the $x_{i}, s_{i}$ components of the point $P_{i+1}$ in the local frame at point $P_{i}$. by

The angle $\Phi_{i+}$ is the angle of the $\mathscr{P}_{i+1}$ frame relative to the $\mathscr{P}_{i}$ frame. It is given

$$
\begin{aligned}
\Phi_{i+} & =\Theta_{i+1}-\Theta_{i}(i=0, N-1) \\
& =-2 \pi-\Theta_{N}(i=N) .
\end{aligned}
$$

For a large proton accelerator with the angle conventions used, it will be a very small negative angle. This angle and the trigonometric functions $\sin \Phi_{i+}, \cos \Phi_{i+}$, and $\tan \Phi_{i+}$ are evaluated and stored in advance of tracking. The other quantities needed are

$$
\begin{aligned}
& \mathscr{L}_{i+}=\left[\left(Z_{i+1}-Z_{i}\right)^{2}+\left(X_{i+1}-X_{i}\right)^{2}\right]^{1 / 2}, \\
& \mathscr{S}_{i+}=\mathscr{L}_{i+} \cos \left(\Theta_{i+}-\Theta_{i}\right), \quad(i=0, N), \\
& \mathscr{L}_{i+}=\mathscr{L}_{i+} \sin \left(\Theta_{i+}-\Theta_{i}\right) .
\end{aligned}
$$




\section{MECHANICAL TRANSLATION OF A REALISTIC LATTICE INTO AN EQUIVALENT THIN ELEMENT LATTICE}

After this translation, the only elements appearing in the lattice description are multipoles whose maximum orders are flagged to avoid unnecessary calculations with zero elements.

\subsection{Translation}

The program TEAPOT reads a lattice in the standard input format. ${ }^{1}$ Magnetic elements in this description may be either thin (multipoles) or thick (quadrupoles, sector bends, sextupoles, octupoles). The program takes all thick elements and replaces them with a corresponding thin version.

The transfer matrix for a quadrupole is

$$
\left(\begin{array}{cc}
\cos \sqrt{\frac{L}{f}} & \sqrt{L f} \sin \sqrt{\frac{L}{f}} \\
-\sqrt{\frac{1}{L f}} \sin \sqrt{\frac{L}{f}} & \cos \sqrt{\frac{L}{f}}
\end{array}\right),
$$

where $f=L k_{1}$. If we expand in $L / f$, we get

$$
\left(\begin{array}{cc}
1-\frac{L}{2} f+\cdots & L\left(1-\frac{L}{6 f}+\cdots\right) \\
-\frac{1}{f}\left(1-\frac{L}{6 f}+\cdots\right) & 1-\frac{L}{2 f}+\cdots
\end{array}\right)
$$

A thin quadrupole has $L=0, f$ finite, so its matrix is

$$
\left(\begin{array}{rr}
1 & 0 \\
-\frac{1}{f} & 1
\end{array}\right) \text {. }
$$

If we approximate a quadrupole of length $L$ by a thin quadrupole at its center, we get

$$
\left(\begin{array}{cc}
1 & L / 2 \\
0 & 1
\end{array}\right)\left(\begin{array}{cc}
1 & 0 \\
-1 / f & 1
\end{array}\right)\left(\begin{array}{cc}
1 & L / 2 \\
0 & 1
\end{array}\right)=\left(\begin{array}{cc}
1-L / 2 f & L(1-L / 4 f) \\
-1 / f & 1-L / 2 f
\end{array}\right)
$$

Comparing this matrix to that of Eq. (20), we see that for $L / f$ small, or to first order in $L / f$, we have a reasonable approximation. The biggest errors we make will be for the off-diagonal terms.

If $L / f$ is sufficiently small, a single thin lens is a good approximation to the thick lens. If we need more accuracy, we can replace the thick lens by several thin lenses, which give higher-order terms in $L / f$ in the transfer matrix. Optimal spacings and strengths for these thin elements are given in Ref. 5. In the program 


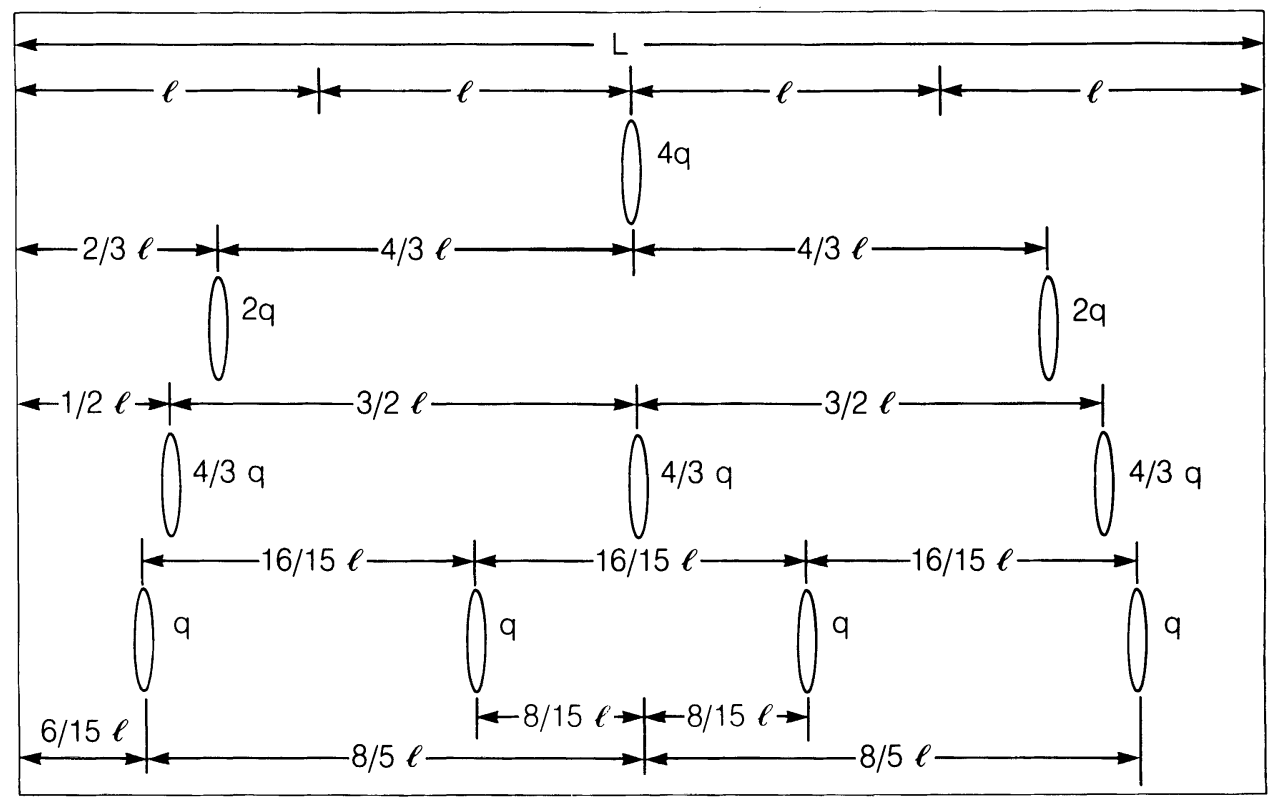

FIGURE 3 "Best fit" representation of a thick lens by $n$ thin lenses. $K L=4 q=1 / f$. In TEAPOT at present, only the top $(n=1)$ and bottom $(n=4)$ cases are used. Exact dimensions and strengths must be maintained. See Ref. 5 .

TEAPOT, quadrupoles are treated as single thin lenses unless their definition includes the statement TYPE $=I R$, in which case they are split into four thin quadrupoles, each having a strength one-quarter that of the original thick quadrupole. The spacings are indicated in Fig. 3, taken from Ref. 5. For an ideal thin quadrupole the coefficient $b$ and, if skewed, $a$ in Eq. (1) are nonvanishing, but all other elements are zero.

Only sector bends are allowed bends in TEAPOT. They are treated as thin wedge dipoles with horizontal focusing. The full thick dipole matrix is

$$
\left(\begin{array}{cc}
\cos \alpha & \rho \sin \alpha \\
-\frac{1}{\rho} \sin \alpha & \cos \alpha
\end{array}\right),
$$

where $\alpha$ is the bend angle and $\rho$ is the bend radius. The thin dipole matrix in the horizontal plane is

$$
\left(\begin{array}{cc}
1 & 0 \\
-\frac{\sin \alpha}{\rho} & 1
\end{array}\right)
$$

Wedge dipoles have unit matrix in the vertical plane. To represent the dipole in the form given by Eq. (1), a special quad of strength $b_{01}$, which focuses only in the horizontal plane, is introduced. As is well-known, the restriction to sector 
bends can be overcome by placing thin quadrupoles at the entrance and exit planes to account for edge-focusing.

\subsection{Tuning of Lattice Parameters}

Since the thin-lens matrix is only an approximation to the correct thick-lens one, when a lattice is converted to a thin-lens equivalent, the Twiss parameters may not be sufficiently accurate. In particular, the tune of the thin-lens machine may be incorrect. TEAPOT allows the user to fit the horizontal and vertical tunes of the thin-lens machine to specified accuracy by varying two families of quadrupoles. This gives improved accuracy in $\beta$ and $\alpha$ also.

\subsection{Example Lattices}

Two lattices will be used as examples of the Twiss analysis and fitting of thin-lens approximations to real lattices. First, a lattice made entirely of FODO cells, of a type similar to that used in the SSC test lattices, was analyzed. The lattice consists of $40960^{\circ}$ cells. A comparison of the lattice before and after fitting the quadrupole strength with the corresponding thick-lens lattice is given in Table I.

The second lattice analyzed was the SSC clustered-IR test lattice. This lattice includes six interaction regions, four on one side of the racetrack and two on the other, along with two utility sections. The nominal phase advance is $60^{\circ}$ per cell,

TABLE I

FODO Lattice Parameters

\begin{tabular}{lrrr}
\hline & $\begin{array}{c}\text { Thin lens } \\
\text { before fit }\end{array}$ & $\begin{array}{c}\text { Thin lens } \\
\text { after fit }\end{array}$ & Thick lens \\
\hline$\beta_{x}$ & 323.031 & 323.567 & 324.356 \\
$\beta_{y}$ & 108.999 & 109.885 & 109.624 \\
$Q_{x}$ & 68.553 & 68.185 & 68.185 \\
$Q_{y}$ & 68.563 & 68.195 & 68.195 \\
\hline
\end{tabular}

TABLE II

Values for $L / f$ for Clustered-IR

Lattice

\begin{tabular}{lc}
\hline Quad & $L / f$ \\
\hline Arc quad & 0.05 \\
IR quad 1 & 0.17 \\
IR quad 2 & 0.43 \\
IR quad 3 & 0.51 \\
IR quad 4 & 0.06 \\
IR quad 5 & 0.31 \\
IR quad 6 & 0.02 \\
Phase trombone & 0.07 \\
\hline
\end{tabular}


and the interaction regions include phase trombones and dispersion suppressors. The main arc quad strengths were varied to fit the tunes to the desired values. The strengths of all other quadrupoles were the same as those in the thick-lens lattice, and all the interaction-region and phase-trombone quadrupoles, of type IR, were split by the program into four thin quadrupoles. Table II gives $L / f$ for

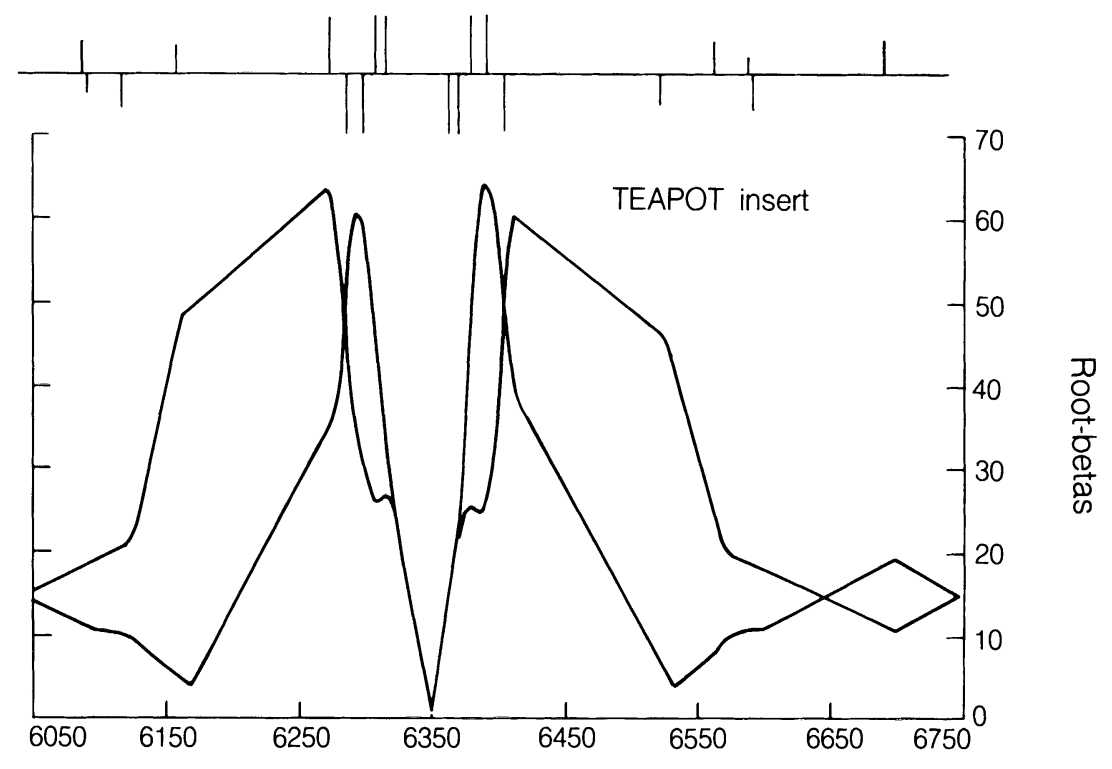

Path length
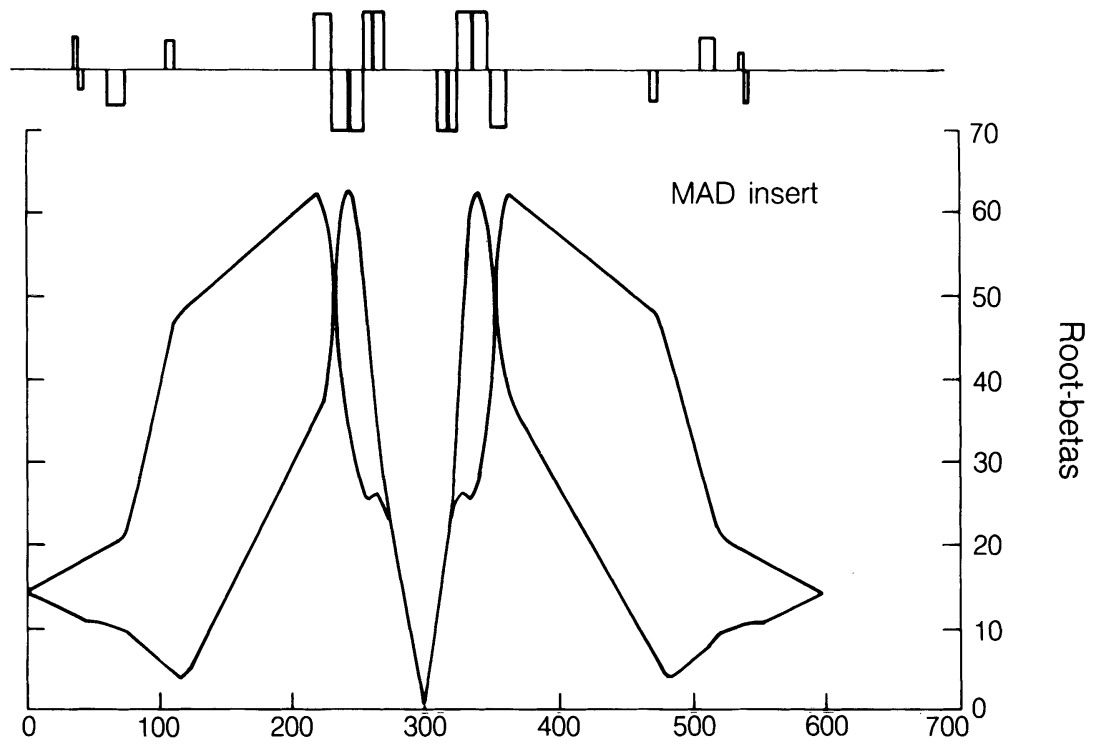

Path length

FIGURE 4 Beta functions for one interaction region, computed by (a) TEAPOT and (b) MAD. 
TABLE III

Beta Values for the Six Interaction Regions of a Clustered-IR lattice

\begin{tabular}{ccc}
\hline IR & $\beta_{x}^{*}$ & $\beta_{y}^{*}$ \\
\hline 1 & 1.013 & 0.889 \\
2 & 0.965 & 1.080 \\
3 & 1.003 & 0.901 \\
4 & 0.945 & 0.968 \\
5 & 1.023 & 1.006 \\
6 & 0.957 & 1.086 \\
\hline
\end{tabular}

various quadrupoles in the lattice. The bend angle of the dipoles (five per half cell) is $1.5 \mathrm{mr}$.

Figures $4 \mathrm{a}$ from TEAPOT and $4 \mathrm{~b}$ from MAD show the beta functions in the interaction region only, for that IR having the worst agreement between TEAPOT and a thick-lens optics program. Table III lists $\beta^{*}$ for all six interaction regions. For the thick-lens lattice, all entries are $1.0 \mathrm{~m}$. If better accuracy were required, the phase-trombone quads could be fit to give the correct phase and advance, thus decreasing the beta mismatch there. When the IR region alone is fit, the values are $\beta_{x}^{*}=0.981, \beta_{y}^{*}=0.991$, and the phase advance across the IRs are $1.053,1.054$ (instead of $1.054,1.055$ ). On the scale of Fig. 4 , the thick- and thin-lens plots are indistinguishable.

\section{PARTICLE TRACKING}

Initially the particle displacements $x, y, \delta$ are given at the start of the lattice. Let us assume that the trajectory has been tracked up to just before the $i$ th multipole, with the coordinates in the $\mathscr{P}_{i}$ frame being $x_{i}, y_{i}, s_{i}$. The velocity components just before the multipole, also in frame $\mathscr{P}_{i}$, are $v_{x i}, v_{y i}, v_{s i}$ with magnitude $v_{i}$. To advance to the point of having the corresponding information at plane $\mathscr{P}_{i+1}$, three calculations must be performed. They are deflection by the multipole at $\mathscr{P}_{i}$, propagation through the drift space, and coordinate transformation into $\mathscr{P}_{i+1}$ coordinates. These are described in the following three sections.

\subsection{Deflection in a Multipole}

According to Eq. (7), the transverse velocity components just after the multipole at $P_{i}$ are given by

$$
\begin{aligned}
& v_{x}=v_{x i}+\frac{1}{1+\delta} F_{x} v_{i} \\
& v_{y}=v_{y i}+\frac{1}{1+\delta} F_{y} v_{i}
\end{aligned}
$$


where

$$
\begin{aligned}
& F_{x}=-\frac{B_{y} L}{p_{0} / e}=-\tilde{B}_{y}, \\
& F_{y}=\frac{B_{x} L}{p_{0} / e}=\tilde{B}_{x} .
\end{aligned}
$$

Also $\delta=\delta_{i}$, and, using Eq. (9),

$$
v_{s}=\left(v_{i}^{2}-v_{x}^{2}-v_{y}^{2}\right)^{1 / 2} .
$$

These remain valid throughout the region from $P_{i}$ to $P_{i+1}$. As mentioned following Eq. (3), $\delta$ is not assumed to be small, and all these equations are exact.

The field integrals are evaluated using Eq. (1). We use an efficient algorithm copied from $\mathrm{MAD}^{4}$ (with some modification) for evaluating them. Recopying Eq. (1) and, for simplicity, setting $\Delta x=\Delta y=0$, we have

$$
\begin{aligned}
\tilde{B}_{y}+i \tilde{B}_{x} & =\sum_{n=0}^{M}\left(\tilde{b}_{n}+i \tilde{a}_{n}\right)(x+i y)^{n} \\
& =\sum_{n=0}^{M}\left(\tilde{b}_{n}+i \tilde{a}_{n}\right)\left(R_{n}+i I_{n}\right),
\end{aligned}
$$

where

$$
\begin{aligned}
R_{n} & =\operatorname{Re} z^{n}, \\
I_{n} & =\operatorname{Im} z^{n}, \\
z & =x+i y .
\end{aligned}
$$

For illustration the values for $n \leq M=2$ are

$$
\begin{array}{ll}
R_{0}=1, & I_{0}=0, \\
R_{1}=x, & I_{1}=y, \\
R_{2}=x^{2}-y^{2}, & I_{2}=2 x y .
\end{array}
$$

The velocity increments appearing in Eq. (25) are determined by

$$
\begin{aligned}
& F_{x}=\sum_{n=0}^{M}\left(-\tilde{b}_{n} R_{n}+\tilde{a}_{n} I_{n}\right), \\
& F_{y}=\sum_{n=0}^{M}\left(\tilde{b}_{n} I_{n}+\tilde{a}_{n} R_{n}\right) .
\end{aligned}
$$

A pure erect quadrupole is described by

$$
\begin{aligned}
& F_{x}=-\tilde{b_{1}} x, \\
& F_{y}=\tilde{b}_{1} y .
\end{aligned}
$$


A pure erect sextupole is described by

$$
\begin{aligned}
& F_{x}=-\tilde{b}_{2}\left(x^{2}-y^{2}\right), \\
& F_{y}=2 \tilde{b}_{2} x y .
\end{aligned}
$$

One algorithm for the evaluation of the fields is to work out all values of $R_{n}$ and $I_{n}$ for $n=0$ to $M$, using the starting values for $R_{0}$ and $I_{0}$ given by Eqs. (30) and the obvious recursion relations

$$
\begin{aligned}
R_{n+1} & =x R_{n}-y I_{n}, \\
I_{n+1} & =y R_{n}+x I_{n},
\end{aligned}
$$

and then to use Eqs. (31). But the algorithm mentioned previously is faster. It is Horner's method applied to a complex power series of terms $z^{n}$ with complex coefficients

$$
\begin{gathered}
C_{n}=\tilde{b}_{n}+i \tilde{a}_{n}, \\
\tilde{B}_{y}+i \tilde{B}_{x}=\sum_{n=0}^{M} C_{n} z^{n}=\cdots+\left\{C_{M-3}+\left[C_{M-2}+\left(C_{M-1}+C_{M} z\right)\right] z\right\} z \cdots
\end{gathered}
$$

Real and imaginary parts are generated recursively by relations resembling Eqs. (34):

$$
\begin{aligned}
\tilde{B}_{y}= & \tilde{b}_{M}, \\
\tilde{B}_{x}= & \tilde{a}_{M}, \\
\tilde{B}_{y}= & x \tilde{B}_{y}-y \tilde{B}_{x}+\tilde{b}_{M-1}, \\
\tilde{B}_{x}= & y \tilde{B}_{y}+x \tilde{B}_{x}+\tilde{a}_{M-1}, \\
\tilde{B}_{y}= & x \tilde{B}_{y}-y \tilde{B}_{x}+\tilde{b}_{M-2}, \\
\tilde{B}_{x}= & y \tilde{B}_{y}+x \tilde{B}_{x}+\tilde{a}_{M-2}, \\
& \circ \quad \circ \quad \circ \\
-F_{x}= & \tilde{B}_{y}=x \tilde{B}_{y}-y \tilde{B}_{x}+\tilde{b}_{0}, \\
F_{y}= & \tilde{B}_{x}=y \tilde{B}_{y}+x \tilde{B}_{x}+\tilde{a}_{0} .
\end{aligned}
$$

There are, altogether, $4 M$ multiplications.

\subsection{Propagation Through the Drift Region}

We are working in the $x_{i}, y_{i}, s_{i}$ coordinate system. The geometry is illustrated in Fig. 5. Again the signs are confusing. It appears that desirable properties, namely, that the charge be positive, that the dipole field be positive upwards, and that $x$ be positive outward, as well as the other MAD conversions, lead to these negative angles. 


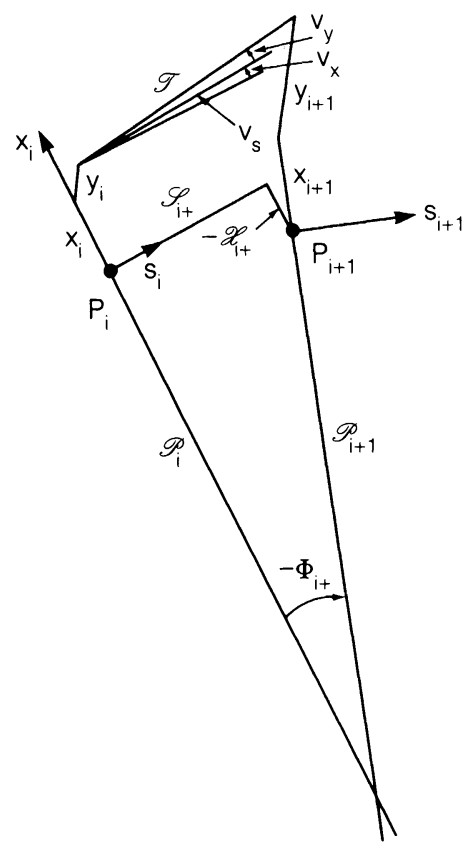

FIGURE 5 The geometry for straight line propagation through a drift space.

The equation of the trajectory $\mathscr{T}$ is

$$
\begin{aligned}
& x-x_{i}=\frac{v_{x}}{v_{s}} s, \\
& y-y_{i}=\frac{v_{y}}{v_{s}} s .
\end{aligned}
$$

The equation of the multipole plane $\mathscr{P}_{i+1}$ is

$$
s-\mathscr{Y}_{i+}=-\tan \Phi_{i+}\left(x-\mathscr{X}_{i+}\right)
$$

One finds the intersection $x_{i+}, y_{i+}, s_{i+}$ of line $\mathscr{T}$ with plane $\mathscr{P}_{i+1}$ to be given by

$$
\begin{aligned}
& x_{i+}=\frac{x_{i}+\frac{v_{x}}{v_{s}}\left(\mathscr{Y}_{i+}+\mathscr{X}_{i+} \tan \Phi_{i+}\right)}{1+\frac{v_{x}}{v_{s}} \tan \Phi_{i+}}, \\
& s_{i+}=\mathscr{S}_{i+}-\tan \Phi_{i+}\left(x_{i+}-\mathscr{X}_{i+}\right), \\
& y_{i+}=y_{i}+\frac{v_{y}}{v_{s}} s_{i+} .
\end{aligned}
$$


The displacement vector from $P_{i+1}$ to this point is given by

$$
\begin{aligned}
x_{i+}-\mathscr{X}_{i+} & =\frac{x_{i}-\mathscr{X}_{i+}+\frac{v_{x}}{v_{s}} \mathscr{S}_{i+}}{1+\frac{v_{x}}{v_{s}} \tan \Phi_{i+}}, \\
s_{i+}-\mathscr{Y}_{i+} & =-\tan \Phi_{i+}\left(x_{i+}-\mathscr{X}_{i+}\right), \\
y_{i+} & =y_{i}+\frac{v_{y}}{v_{s}} \frac{\mathscr{S}_{i+}-\tan \Phi_{i+}\left(x_{i}-\mathscr{X}_{i+}\right)}{1+\frac{v_{x}}{v_{s}} \tan \Phi_{i+}} .
\end{aligned}
$$

\subsection{Rotation into the Next Frame}

The components $x_{i+1}, y_{i+1}$ of the vector given in Eqs. (41) are obtained from

$$
\begin{aligned}
x_{i+1} & =\cos \Phi_{i+}\left(x_{i+}-\mathscr{X}_{i+}\right)-\sin \Phi_{i+}\left(s_{i+}-\mathscr{S}_{i+}\right) \\
& =\left(\cos \Phi_{i+}+\sin \Phi_{i+} \tan \Phi_{i+}\right)\left(x_{i+}-\mathscr{X}_{i+}\right) .
\end{aligned}
$$

Finally we group the equations in the form in which they are actually used in the program

$$
\begin{aligned}
& x_{i+1}=\frac{1}{\cos \Phi_{i+}} \frac{x_{i}-\mathscr{X}_{i+}+\frac{v_{x}}{v_{s}} \mathscr{S}_{i+}}{1+\frac{v_{x}}{v_{s}} \tan \Phi_{i+}}, \\
& y_{i+1}=y_{i}+\frac{v_{y}}{v_{s}} \frac{\mathscr{S}_{i+}-\tan \Phi_{i+}\left(x_{i}-\mathscr{X}_{i+}\right)}{1+\frac{v_{x}}{v_{s}} \tan \Phi_{i+}}, \\
& s_{i+1}=0 .
\end{aligned}
$$

The velocity components must also be transformed:

$$
\begin{aligned}
& v_{x(i+1)}=v_{x} \cos \Phi_{i+}-v_{s} \sin \Phi_{i+}, \\
& v_{y(i+1)}=v_{y}, \\
& v_{s(i+1)}=v_{x} \sin \Phi_{i+}+v_{s} \cos \Phi_{i+}
\end{aligned}
$$

The equations which have to be evaluated in tracking one particle from one multipole to the next have been enclosed in boxes. In a linear transfer matrix representation, $v_{x}$ and $v_{y}$ would be proportional to $d x / d s$ and $d y / d s$, respectively. Realizing that $\Phi_{i+}$ is a very small angle, one can recognize the leading terms of Eqs. (25) and (43) as essentially these linear transformations. There are, 
however, nonlinear corrections such as the denominator factors, even for pure drifts or pure quadrupoles. Furthermore, in the transformation $x_{i}, v_{x i} \rightarrow x_{i+1}$, $v_{x(i+1)}$, the Jacobian is not unity, and hence $\left(x, v_{x}\right)$ phase-space densities are not conserved. This can be seen to be true for passage through any thin element, since the angle of deflection is a function (very weak, of course) of the angle of incidence.

The statement that this tracking is exact should probably be qualified. The only forces acting are calculated using the Eq. (1), and the resulting motion is calculated exactly in three-dimensional space for all amplitudes $x, y$. But when $x$ and/or $y$ is too large, the expansion of Eq. (1) necessarily becomes unphysical. That is, the fields which it yields are not the same as the fields in the realizable magnet which it is supposed to represent. This breakdown is somewhat academic, since, for regions which particles actually visit, Eq. (1) contains the best available information about the fields. A related observation is that the amplitudes $x, y$ actually encountered during tracking are numerically small, even though, in principle, they could be comparable in magnitude with the gross dimensions of the accelerator. This is because they are always referred to the local coordinate frame. TEAPOT also neglects fringe fields; that is, it sets fringe fields to zero. For fields in vacuum, this is unphysical, since field discontinuities require current sheets. To maintain the claim that the machine which TEAPOT tracks is "physical," such current sheets must be assumed to be present.

\section{EXTRACTION OF LATTICE FUNCTIONS FROM TRACKING OUTPUT}

One of the purposes of TEAPOT is to obtain lattice functions in the presence of errors. Since the program does not work with transfer matrices, it is necessary to extract them from tracking data. Procedures for doing this and then calculating lattice functions are described in this section. The equations here are formulated in somewhat greater generality than they are presently implemented in TEAPOT, which assumes that the lattice is sufficiently decoupled that, for one turn, treating the horizontal and vertical motion as independent is adequate. In Section 6, the TEAPOT decoupling instruction which accomplishes this (for sufficiently small assumed errors) is described.

\subsection{A Polynomial Fit}

The claims made elsewhere in this paper about exactness must be greatly tempered in this section, as questions of round-off are clearly important here, and they have not been thoroughly investigated. In TRANSPORT notation,

$$
x=\left(x_{1}, x_{2} \cdots x_{6}\right)=\left(x, x^{\prime}, y, y^{\prime}, x_{5}, \delta\right),
$$

where $x_{5}$ plays no role for our purposes. To facilitate separate discussion of momentum dependence, we define two types of index

$$
\begin{array}{r}
i, j, k, \ldots \text { range over } 1,2,3,4,6 \\
\alpha, \beta, \gamma, \ldots \text { range over } 1,2,3,4
\end{array}
$$


The effect of tracking is to calculate functions $f(x)$, where $f$ can stand for any one of the output coordinates $x_{\gamma}$ (out). Any such function can be approximated by a polynomial

$$
\begin{aligned}
f(X) & =Q+\sum_{j} R_{j} x_{j}+\sum_{j} T_{j j} x_{j}^{2}+\sum_{j \neq k} T_{j k} x_{j} x_{k} \\
& =Q+R+T_{1}+T_{2} .
\end{aligned}
$$

As defined, $T_{j k}$ is symmetric (a slight variation from TRANSPORT notation):

$$
T_{j k}=T_{k j} \text {. }
$$

The coefficients $Q, R$, and $T$ acquire an extra subscript $\gamma$ when we specialize to a particular output coordinate $x_{\gamma}$. To obtain these coefficients in general, one must track starting from 31 different initial conditions. (In practice, at this stage, TEAPOT needs to track only 11 rays.)

Tracking from the origin, one obtains

$$
Q=f(0) \text {. }
$$

Then, setting all $x_{i}$ except $x_{j}$ to zero, one gets

$$
[-Q+f(X)]_{\substack{i=0 \\ i \neq j}}=R_{j} x_{j}+T_{j j} x_{j}^{2}
$$

Evaluating this for appropriately small starting values $\pm x_{j}$, one obtains $R_{j}$ from the difference and $T_{j j}$ from the sum. One then gets $T_{j k}$ by zeroing all but $x_{j}$ and $x_{k}$ in the quantity $f(X)-Q-R-T_{1}$. Except for the global decoupling, TEAPOT does not use, and does not calculate, the off-diagonal elements coupling $x$ and $y$, even though that would be straightforward. Also, the elements with $j$ or $k$ equal to six will only be needed in a slightly revised expansion described below in Section 5.3.

The quantity $x_{j}$ above is a typical value chosen to be large enough to ameliorate the above-mentioned round-off problem, but small enough to avoid pollution from nonlinear terms. With care in this regard, lattice functions, including chromaticities, are obtained that are in excellent agreement with other determinations, accuracy invariably being better than $2 \%$. This accuracy is, however, greatly inferior to the tracking accuracy to be described in Section 7.

\subsection{Closed-Orbit Determination}

Setting $\delta$ to zero and interpreting $f(X)$ as the on-momentum closed-orbit displacement $X^{(0)}=\left(x_{\gamma}^{(0)}\right)$, one obtains

$$
x_{\gamma}^{(0)}=Q_{\gamma}+\sum_{\alpha} R_{\gamma \alpha} x_{\alpha}^{(0)}+\sum_{\alpha, \beta} T_{\gamma \alpha \beta} x_{\alpha}^{(0)} x_{\beta}^{(0)},
$$

which is the condition that $X^{(0)}$ replicate itself after one turn. To avoid solving these nonlinear equations, there is an approximate, two-step procedure which starts by neglecting the quadratic terms which are presumably small. This yields the matrix equation

$$
M X_{0}^{(0)}=Q,
$$


where $Q \equiv\left(Q_{\gamma}\right)$ and

$$
M=\left(\begin{array}{cccc}
1-R_{11} & -R_{12} & -R_{13} & -R_{14} \\
-R_{21} & 1-R_{22} & -R_{23} & -R_{24} \\
-R_{31} & -R_{32} & 1-R_{33} & -R_{34} \\
-R_{41} & -R_{42} & -R_{43} & 1-R_{44}
\end{array}\right) .
$$

An improved solution $X^{(0)}$ is then obtained from $X_{0}^{(0)}$ by the replacement in Eq. (52) of

$$
Q_{\gamma} \rightarrow Q_{\gamma}+\sum_{\alpha, \beta} T_{\gamma \alpha \beta} x_{\alpha}^{(0)} x_{\beta}^{(0)}
$$

Since, at present, TEAPOT assumes that block off-diagonal elements vanish (how this is accomplished is described in Section 5.5), $M$ can be inverted explicitly:

$$
\left(\begin{array}{cc}
1-R_{11} & -R_{12} \\
-R_{21} & 1-R_{22}
\end{array}\right)^{-1}=\left(\begin{array}{cc}
1-R_{22} & R_{12} \\
R_{21} & 1-R_{11}
\end{array}\right) /\left|\begin{array}{cc}
1-R_{11} & -R_{12} \\
-R_{21} & 1-R_{22}
\end{array}\right|
$$

$X^{(0)}$ is then tracked around the machine until convergence is achieved.

\subsection{Transfer Matrices Relative to the Closed Orbit}

When operating an actual machine, measurable quantities like tunes and chromaticities relate to the actual closed orbit as it is determined by errors and misalignments. It is therefore useful to work out transfer matrices $\bar{R}$ and $\bar{T}$ relative to the closed orbit. Define a displacement vector $\bar{X}=\left(\bar{x}_{\gamma}\right)$ relative to the closed orbit by

$$
x_{\gamma}=x_{\gamma}^{(0)}+\bar{x}_{\gamma} .
$$

Equation (47) is replaced by

$$
\bar{x}_{\gamma}(\text { out })=\sum_{j} \bar{R}_{j} \bar{x}_{j}+\sum_{j} \bar{T}_{j j} \bar{x}_{j}^{2}+\sum_{j \neq k} \bar{T}_{j k} \bar{x}_{j} \bar{x}_{k} .
$$

The elements $\bar{R}_{j}$ and $\bar{T}_{j k}$ can be obtained as in Section 5.1 by tracking 17 new special rays which are displaced appropriately from the closed orbit. Note that the feed-down effects from higher multipoles are incorporated by doing this.

The perturbed tunes can be obtained from $\bar{R}$. Also, the same 17 rays can be used to calculate the perturbed beta functions at any point of interest in the lattice. The formulas are

and similarly for $y$.

$$
\begin{aligned}
\cos 2 \pi \bar{v}_{x} & =\frac{\bar{R}_{11}+\bar{R}_{22}}{2}, \\
\bar{\beta}_{x} & =\frac{\bar{R}_{12}}{\sin 2 \pi \bar{v}_{x}}, \\
\bar{\alpha}_{x} & =\frac{\bar{R}_{11}-\bar{R}_{22}}{2 \sin 2 \pi \bar{v}_{x}},
\end{aligned}
$$




\subsection{Momentum Dependence of the Perturbed Lattice}

There is a well-known prescription ${ }^{6,7}$ for obtaining dispersion functions and chromaticities from the matrices $\bar{R}$ and $\bar{T}$. To find the horizontal dispersion, one proceeds in two steps (like those in Section 5.2), with the result

$$
\begin{gathered}
\eta_{x}=\eta_{0 x}+\delta \eta_{1 x}, \\
\left(\begin{array}{l}
\eta_{0 x} \\
\eta_{0 x}^{\prime}
\end{array}\right)=S\left(\begin{array}{l}
\bar{R}_{16} \\
\bar{R}_{26}
\end{array}\right)=\frac{1}{4 \sin ^{2}\left[\pi v_{x}(0)\right]}\left(\begin{array}{cc}
1-\bar{R}_{22} & \bar{R}_{12} \\
\bar{R}_{21} & 1-\bar{R}_{11}
\end{array}\right)\left(\begin{array}{l}
\bar{R}_{16} \\
\bar{R}_{26}
\end{array}\right),
\end{gathered}
$$

and

$$
\left(\begin{array}{c}
\eta_{1 x} \\
\eta_{1 x}^{\prime}
\end{array}\right)=S\left(\begin{array}{l}
\bar{T}_{111} \eta_{0}^{2}+2 \bar{T}_{112} \eta_{0} \eta_{0}^{\prime}+\bar{T}_{122} \eta_{0}^{\prime 2}+2 \bar{T}_{116} \eta_{0}+2 \bar{T}_{126} \eta_{0}^{\prime}+\bar{T}_{166} \\
\bar{T}_{211} \eta_{0}^{2}+2 \bar{T}_{212} \eta_{0} \eta_{0}^{\prime}+\bar{T}_{222} \eta_{0}^{\prime 2}+2 \bar{T}_{216} \eta_{0}+2 \bar{T}_{226} \eta_{0}^{\prime}+\bar{T}_{266}
\end{array}\right)
$$

There are similar formulas for vertical dispersion.

One can define a transfer matrix $\bar{R}^{(\delta)}$ relative to an off-momentum closed orbit which is displaced from the perturbed closed orbit (itself displaced from the nominal closed orbit). From Ref. 6 , the formulas are

$$
\begin{aligned}
& \bar{R}_{11}^{(\delta)}=\bar{R}_{11}+2\left(\bar{T}_{111} \eta_{x}+\bar{T}_{112} \eta_{x}^{\prime}+\bar{T}_{116}\right) \delta, \\
& \bar{R}_{12}^{(\delta)}=\bar{R}_{12}+2\left(\bar{T}_{122} \eta_{x}^{\prime}+\bar{T}_{112} \eta_{x}+\bar{T}_{126}\right) \delta, \\
& \bar{R}_{21}^{(\delta)}=\bar{R}_{21}+2\left(\bar{T}_{211} \eta_{x}+\bar{T}_{212} \eta_{x}^{\prime}+\bar{T}_{216}\right) \delta, \\
& \bar{R}_{22}^{(\delta)}=\bar{R}_{22}+\left(2 \bar{T}_{222} \eta_{x}^{\prime}+\bar{T}_{212} \eta_{x}+\bar{T}_{226}\right) \delta .
\end{aligned}
$$

The dependence of tune $v_{x}$ on momentum can then be obtained from Eq. (58) with $\bar{R}_{11}$ and $\bar{R}_{22}$ generalized to $\bar{R}_{11}^{(\delta)}$ and $\bar{R}_{22}^{(\delta)}$. To obtain the horizontal chromaticity, this equation is to be differentiated with respect to $\delta$.

\subsection{Coupling}

Global decoupling of the machine involves zeroing the four matrix elements $R_{31}$, $R_{32}, R_{41}$, and $R_{42}$. This can be accomplished by adjusting the strengths of four skew quadrupole families with the DECOUPLE command described in Section 6.

\section{SOME COMMANDS RECOGNIZED BY TEAPOT}

In this section, the majority of the commands understood by TEAPOT and the operations that they elicit are briefly described. Detailed instructions are contained in a manual available from the authors.

TEAPOT is a tracking code which treats all elements (aside from drifts) as thin elements. A lattice described in Standard Input Format is read in, and thick elements are mechanically converted into thin elements. A Twiss analysis can be performed using a thin-lens matrix representation of the machine. From this the tunes can be readjusted (often a small or negligible shift, especially if specially sensitive quadrupoles are broken into shorter lengths). Magnet errors and 
misalignments can be added to elements, and the resulting lattice can be tracked exactly. As described previously, a full Twiss analysis in the presence of these errors is available, which uses tracking to derive the transfer matrices for the machine. The machine can be decoupled using skew quads, the tunes can be readjusted, and the chromaticity can be fit in the presence of errors. In short, many of the tuning operations routine in normal accelerator operation can be modeled.

These operations are performed in response to the following commands:

TWISS. Invokes a Twiss analysis using a matrix representation. This is useful to provide a reference by which the faithfulness of the thin-element lattice can be judged.

TUNE. Compensates for the tune shifts caused by the conversion from thick to thin elements. Naturally, trim quadrupoles must be present in the lattice for this purpose.

MAKETHIN. Invokes the mechanical conversion from thick to thin elements and creates the data structures which represent the thin-lens machine in a form appropriate for tracking. This includes finding the reference orbit.

WRITEFILE. Writes a file fully describing the thin-lens machine after all desired tuning has been performed and before tracking has been done, so that in subsequent runs the already tuned lattice can be recovered using READFILE.

ERRORS. Assigns rms multipole and position errors including a random seed and the possibility of setting cuts.

TRACK. Assigns initial coordinates and other attributes to one particle and specifies the number of turns for which it is to be tracked. As many as 1000 particles can be tracked, but there must be one TRACK command for each one. Particle coordinates are recorded each turn.

ANALYSIS. Performs the calculations described in Section 5. It must be performed to provide the information needed to perform the functions DECOUPLE, TUNETHIN, and CHROMFIT, which perform the lattice-tuning operations which their names suggest. Appropriate lattice trim elements must be present for them to work on, and ANALYSIS must be repeated each time if further tuning on top of some completed tuning is to be performed.

\section{COMPARISONS WITH OTHER PROGRAMS}

The purpose of this section is to show the correctness of TEAPOT tracking in contrast to standard codes like RACETRACK and PATRICIA, which treat nonlinear elements as kicks. This will be done using LIEPOT, ${ }^{2}$ to extract sensitive parameters like chromaticity from behavior close to the equilibrium orbit. We would like to express our gratitude to E. Forest for performing these calculations.

As we have pointed out, TEAPOT solves exactly for the thin-lens kick. 
TABLE I

Comparison of MARYLIE and RACETRACK

\begin{tabular}{lccccccc}
\hline & $Q_{x}^{\prime}$ & $Q_{y}^{\prime}$ & $Q_{x}^{\prime \prime}$ & $Q_{y}^{\prime \prime}$ & $a$ & $b \times\left(10^{-2}\right)$ & $c$ \\
\hline MARYLIE & -1.07619 & -1.60531 & 2.77269 & 4.33913 & 0.100320 & 6.00842 & 0.218390 \\
RACETRACK & -3.07006 & -2.16831 & $\mathrm{nc}$ & $\mathrm{nc}$ & 0 & 0 & 0 \\
\hline
\end{tabular}

Dividing Eq. (25) by the total velocity $v_{i}$ gives the correct expression:

$$
\frac{v_{x}}{v_{i}}=\frac{v_{x i}}{v_{i}}+\frac{F_{x}}{1+\delta} .
$$

This is to be compared with the incorrect expression used in ordinary kick codes:

$$
\frac{d x}{d s}=\frac{v_{x}}{v_{s}}=\frac{v_{x i}}{v_{s i}}+\frac{F_{x}}{1+\delta} .
$$

Dragt has noticed that the inadequacy of Eq. (66) is felt mostly in small storage rings. In particular, codes using this formula do not compute the chromaticities correctly. It should also be added that quadrupoles and dipoles are not really "linear." However, if Eq. (66) is used, they become "linear." We will now show that TEAPOT can reproduce the correct results for a machine composed of ideal elements, provided that the dipoles are split into a sufficient number of thin elements. This is what we mean when we claim that TEAPOT is a symplectic integrator.

We will use the proton storage ring at Los Alamos, PSR. ${ }^{8}$ The lattice is composed of 10 simple FODO cells with $36^{\circ}$ sector bends. Using the programs MARYLIE,${ }^{9}$ LIEPOT, ${ }^{2}$ and RACETRACK,${ }^{10}$ we have computed the first- and second-order chromaticities and the triplet $(a, b, c)$ which gives the emittancedependent tune shifts, defined by

$$
\left(\begin{array}{c}
\Delta Q_{x} \\
\Delta Q_{y}
\end{array}\right)=\left(\begin{array}{ll}
a & b \\
b & c
\end{array}\right)\left(\begin{array}{l}
\epsilon_{x} \\
\epsilon_{y}
\end{array}\right) .
$$

Table I compares MARYLIE and RACETRACK. In the table, nc means the parameter is not calculated by RACETRACK. As one can see, the agreement is poor. The tunes agree, as they should, since both programs have the same linear matrices. Furthermore, the numbers for $Q_{x}^{\prime}$ and $Q_{y}^{\prime}$ provided by MARYLIE agree very well (to all digits provided) with the numerical results of Dragt (Ref. 8, Table VIII). The results of MARYLIE are the results of algebraic manipulations on the map, and therefore they are exact to computer accuracy.

In Table II, we display the numbers predicted by LIEPOT for the case of one kick $(N=1)$, three kicks $(N=3)$, and one hundred kicks $(N=100)$ per bend, with $4 N$ kicks per quadrupole. In all three cases, we have modified the quadrupole strengths in TEAPOT so that the tunes of the thin-lens machine are correct. The necessary changes in the quadrupole strengths, which decrease with 
TABLE II

Predictions from LIEPOT for Different Numbers of Kicks

\begin{tabular}{rccccccccc}
\hline$N$ & $Q_{x}^{\prime}$ & $Q_{y}^{\prime}$ & $Q_{x}^{\prime \prime}$ & $Q_{y}^{\prime \prime}$ & $a$ & $b\left(\times 10^{-2}\right)$ & $c$ & $\Delta k f / k f$ & $\Delta k q / k q$ \\
\hline 1 & -0.95474 & -1.49223 & 2.51376 & 3.99160 & 0.118593 & 4.752441 & 0.203687 & -0.074 & 0.025 \\
3 & -1.06507 & -1.59461 & 2.74808 & 4.30725 & 0.101683 & 5.891478 & 0.216922 & -0.007 & 0.003 \\
100 & -1.07618 & -1.60531 & 2.77267 & 4.33910 & 0.100321 & 6.008317 & 0.218389 & $1 \times 10^{-5}$ & $1 \times 10^{-6}$ \\
\hline
\end{tabular}

$N$, are listed in the table. As one sees, the nonlinear properties are more-or-less in the right ballpark, even with $N=1$. The horizontal values are the worst ones, which is expected since the bends are horizontal. With $N=3$, the agreement is quite respectable. Finally, we ran the case of $N=100$ for the coup de grâce. Here, the agreement with MARYLIE is good to five digits. Incidentally, for $N=100$, we have checked all the Lie algebraic polynomials of the sixdimensional map to third order. All coefficients agree to five digits.

Finally, one might say that TEAPOT is just another MARYLIE. This is not true, of course, since MARYLIE is exact to third order around the design trajectory, while TEAPOT is exact to all orders, because it treats the multipole kicks exactly.

\section{REFERENCES}

1. D. C. Carey and F. C. Iselin, "A Standard Input Language for Particle Beam and Accelerator Computer Programs," Proc. of the 1984 Summer Study on the Design and Utilization of the Superconducting Supercollider, Snowmass, CO June 1984; D. Douglas, L. Healy, F. C. Iselin, and R. Ryne, 'Report of the Group on a Common Input Format," SSC Aperture Workshop Summary, SSC-TR-2001, Appendix 7 (1984).

2. E. Forest, Particle Accelerators 22, 15 (1987).

3. Report of the Reference Designs Study Group on the Superconducting Super Collider, (U.S. Dept. of Energy, 1984), p. 84.

4. F. C. Iselin, The MAD Program Reference Manual, CERN-LEP-TH/85 (1985).

5. R. Talman, Representation of Thick Quadrupoles by Thin Lenses, SSC-N-33 (1985).

6. S. G. Peggs, Ph.D. Thesis. Cornell University (1981).

7. R. Talman, "Analysis of Non-Linear Accelerator Lattices", in M. Month, Ed., Summer School on High Energy Particle Accelerators (SLAC, 1982), p. 691.

8. A. J. Dragt, Particle Accelerators 12, 205 (1980).

9. A. J. Dragt et al., IEEE Trans. Nucl. Sci. NS-32, 2311 (1985).

10. A. Wrulich, RACETRACK, A Computer Code for the Simulation of Non-linear Particle Motion in Accelerators," DESY 84-026 (1984). 